\title{
New knowledge of intervertebral disc disease
}

\author{
J. BALL
}

From the Department of Rheumatology, University of Manchester

This paper considers firstly some aspects of dorsolumbar disc degeneration, secondly the disc lesions in rheumatoid arthritis and ankylosing spondylitis and disc calcification.

\section{Disc degeneration}

The common form of disc degeneration is agerelated, but some elderly lumbar spines show little or no disc narrowing or osteophyte formation. The nature of this variation in susceptibility is obscure. Studying discs selected to exclude obvious structural damage, Bijlsma and Copius Peereboom (1972) claimed that ageing, as distinct from pathological degeneration, frays the inner part of the annulus fibrosus. The nucleus pulposus assumes a more fibrotic appearance; contains more chondrones; and, perhaps less characteristically, more granular material (age-pigment) thought to be a proteoglycanlipid complex possibly derived from degenerate cells. Biochemical changes associated with aging include some loss of water from the nucleus pulposus and the presence of proteoglycans which are more extractable (suggesting a reduced association with collagen) and have a greater keratan sulphate: chondroitin sulphate ratio (Gower and Pedrini, 1969; Adams et al., 1977). Interestingly, the collagen content of the nucleus pulposus is reported to be unaffected (Naylor et al., 1975) while its hyaluronic acid content is increased (Adams et al., 1977). Whether the precocious occurrence of these biochemical and morphological changes presages the onset of disc degeneration in some cases is unknown.

The variation in susceptibility to disc degeneration in populations has been well documented by Lawrence (1977) who concludes that, while an innate predisposition cannot be excluded, the type and duration of occupational physical stress is clearly an important factor. The forces acting on a disc are complex (Punjabi, 1977). But Farfan (1977), reviewing his group's work in this field, has proposed that disc degeneration in the upper lumbar spine is mainly due to compression overload whereas in the lower lumbar region it is mainly due to torsional injury. It is known (Nachemson, 1975) from direct measurement in human volunteers that when lifting with the spine anteflexed the intradiscal pressure in the lumbar spine may approach that shown by Jayson et al. (1973) by in-vitro experiments to be capable of fracturing the vertebral end-plate of normal discs, leaving the annulus fibrosus intact. Farfan suggests therefore that compression overload in-vivo fractures the vertebral end-plate with consequent escape of fragments of the nucleus pulposus into the vertebral body, thus producing a Schmorl's node. This removes some of the internal support for the annulus fibrosus, which bulges outwards and promotes osteophyte formation. So far as I am aware this sequence has not been directly observed in man. But an association between Schmorl's node and disc degeneration has recently been documented by Hilton et al. (1976), who have systematically examined cadaveric spines between $T 10$ and $S 1$ selected to exclude gross trauma, infection, neoplasia, and metabolic bone disease. The lower dorsal segments were included because, according to Kellgren (1977), low back pain unaccompanied by leg pain may be due to a lesion in the region of the dorsolumbar junction or in the lower lumbar region.

One of our objectives therefore was to map putative algogenic lesions that might be relevant to low back pain. Schmorl's nodes were the first to be studied. They were found to be significantly more

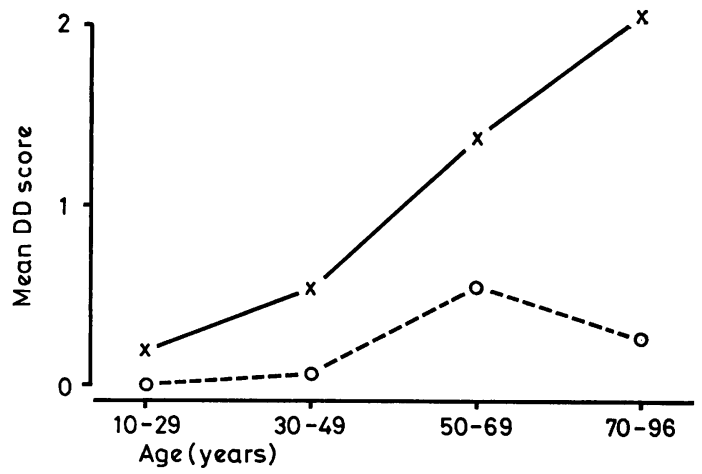

Fig. 1 Mean disc degeneration $(D D)$ score in discs with and without end plate lesion (EPL) (Schmorl's node) in the T 10-L 1 region plotted against age. EPL positive discs $\times-\times$. EPL negative discs $O_{---} \bigcirc$. 
prevalent in the $\mathrm{T} 10-\mathrm{L} 1$ region than at lower levels, and positively correlated with disc degeneration at each of the four segments in the T 10-L1 region. Moreover, in this region the mean disc degeneration score was higher in discs with a Schmorl's node than in those without one at all ages between the second and ninth decades (Fig. 1). In the L 2-L 5 region the overall prevalence of disc degeneration was about the same as in the T 10-L 1 region but Schmorl's nodes were rarely seen and no relationship with disc degeneration was observed. Since Schmorl's nodes were seen in the second decade and were as frequent in those aged under 50 years as in those aged over 50 years it was suggested that Schmorl's nodules originating in early life predispose to disc degeneration and cause its earlier onset.

Whether Schmorl's nodes are always due to compression overload is another matter. It has been shown that the $T 10$ and $T 11$ segments are especially susceptible to torsion (Markolf, 1972). Secondly, Hilton et al. (1976) showed that Schmorl's nodes were unrelated to vertebral bone density, were significantly more common in the lower than the upper vertebral end-plate, and were distributed differently from the vertebral microfractures described by Vernon-Roberts and Pirie (1973). These findings hardly support a traumatic aetiology. Rather they suggest that a Schmorl's node may be a development defect which renders a disc susceptible to physiological compression forces. In any event the causal relationship with disc degeneration is supported by Nachemson's (1960) finding that a disc with a Schmorl's node is mechanically defective.

As regards the second mechanical pathway to disc degeneration, Farfan (1977) has shown experimentally that forced rotation in the lumbar spine injures the outer lamellae of the posterior parts of the annulus fibrosus at sites found in a model system to be zones of stress concentration during rotation. The lamellae are stretched, separated, or even torn from their bony attachments. Repetition of the injury would on theoretical grounds affect deeper lamella with the formation of a deep tear or fissure into which the nucleus pulposus, or fragments thereof, would tend to prolapse. At this point subsequent disc changes would simulate those produced by the other mechanism described above. Since, experimentally, the first signs of injury may appear with as little as three degrees of rotation it seems reasonable to assume that disc damage produced in this way occurs in vivo. The relative rarity of posterior tears in the upper lumbar discs is attributed to their more rounded contour and especially to the more vertical orientation of the facet joints in this region, factors which would increase their resistance to torsion.

In these and other studies of lumbar discs attention has naturally been focussed on fissures and tears in the posterior annulus fibrosus because of their association with disc prolapse. Our own observations (Hilton et al. 1977) on 111 spines confirm the marked predominance of posterior tears in the annulus fibrosus of the L 4 and L 5 discs, especially in those aged under 50 years. However, in this study focal avulsion of the anterior annulus fibrosus was often seen in young adults, and tears in the anterior annulus fibrosus were a conspicuous feature above $\mathrm{L} 2$ in the middle-aged and elderly. Thus age has a greater effect on the anterior annulus fibrosus in the L 2-D 10 region than at lower levels. These studies, which are in progress, suggest that the anterior part of a disc in the dorsolumbar region deserves more attention than it has received in the past, because, theoretically, it could be a source of low back pain.

\section{Disc lesions in rheumatoid arthritis and ankylosing spondylitis}

In rheumatoid arthritis the cervical spine is most severely affected. This is partly because of the neurocentral joints which lie against the lateral borders of cervical discs. These joints, which have a synovial lining externally, are commonly affected. Like synovial joints elsewhere in this disease, involvement is associated with erosion of neighbouring cartilage and bone-in this case the disc-bone border (Ball and Sharp, 1971). Unlike disc degeneration, which has a predilection for the lower cervical spine, a disc at any level may be affected. Similar erosive disc lesions may be seen near the costovertebral joints. In this region they do not lead to severe instability as they characteristically do in the cervical region.

Recent studies of classical ankylosing spondylitis (Ball, 1971; Bywaters, personal communication) indicate that the initial disc lesion is a focal nonspecific inflammation which specifically affects and destroys the attachment of the outer fibres of the annulus fibrosus to the vertebral body just below (or above) the vertebral rim. Similar lesions of ligamentous attachments to bone (entheses) are commonly seen in extra-articular sites and a nonspecific inflammatory enthesopathy is perhaps a pathological hallmark of this disease (Ball, 1971). As in extra-articular sites, the disc lesion heals by direct deposition of reactive bone which initiates the formation of a syndesmophyte, growth of which can cause ankylosis of an otherwise healthy disc. Subsequent calcification and ossification of the disc may well be an effect of immobilisation of the segment and the consequent alteration in stress distribution. 


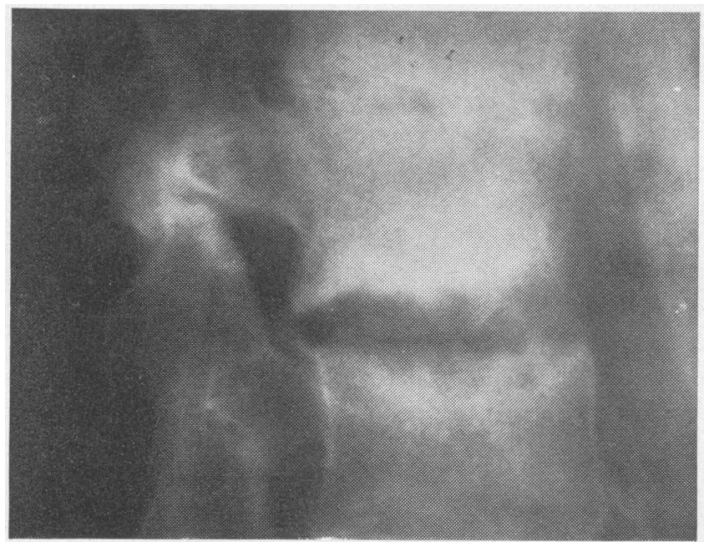

Fig. 2 Ankylosing spondylitis. Destructive lesion of the $L 4 / 5$ disc with fracture of neural arch.

Fig. 3 Ankylosing spondylitis. Diagram of part of macerated spine showing ankylosis at all levels except T $11 / 12$. ankylosing spondylitis are probably due to trauma for the following reasons: (1) they usually occur in the later stages of the disease when the spine is susceptible to minor stress because of the irregular distribution or completeness of bony ankylosis, or both (Fig. 3); (2) they are more often seen in occupationally active spondylitics, and there is radiographic evidence of neural arch fracture in some cases (Fig. 2); (3) there is sequential radiographic evidence that an undisplaced fracture through an ankylosed segment may be followed by changes at the disc-bone border indistinguishable from those of 'spondylodiscitis'; (4) scanty inflammatory cells have been noted in most histological reports although some pathologists regard the overall changes as indistinguishable from those that may be encountered in non-united fractures; and (5) the lesion, unlike the early spondylitic process, responds to rest.

\section{Disc calcification}

That the disc is prone to calcific deposits is well

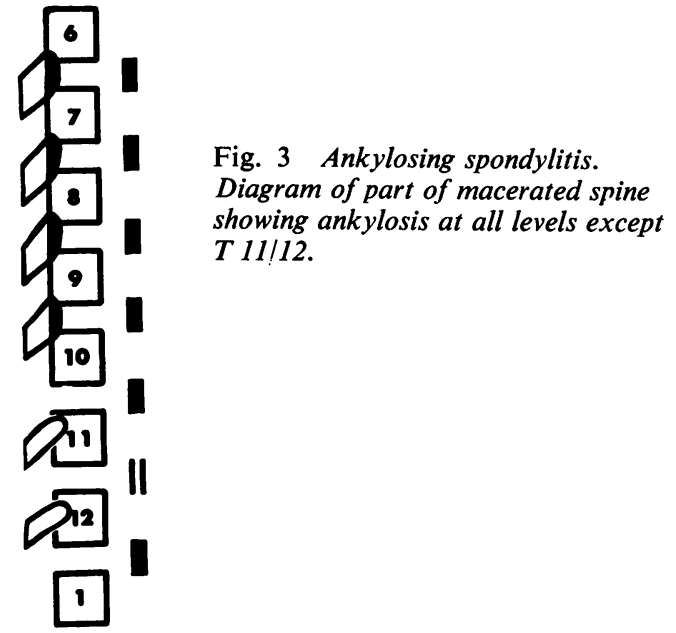

In contrast to this lesion, severe destruction of the whole disc-bone border, commonly referred to as 'spondylodiscitis', may be encountered (Fig. 2). Since there is no evidence that these lesions are due to infection (though they have often been so diagnosed) some think that they represent a severe form of spondylitic inflammation. Others believe they are essentially traumatic - a form of pseudarthrosis. The evidence for both views has recently been critically considered by Cawley et al. (1972) in a report of their own clinicopathological study of this problem. They conclude that severe destructive disc lesions in

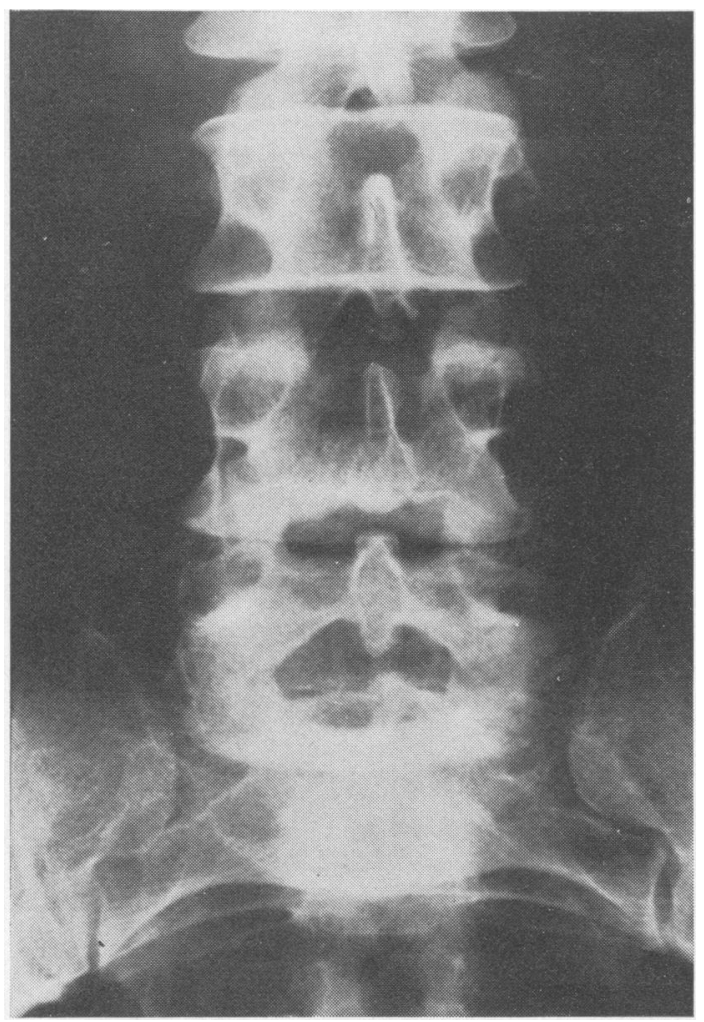

Fig. 4 Narrowing of $L 4 / 5$ disc. 


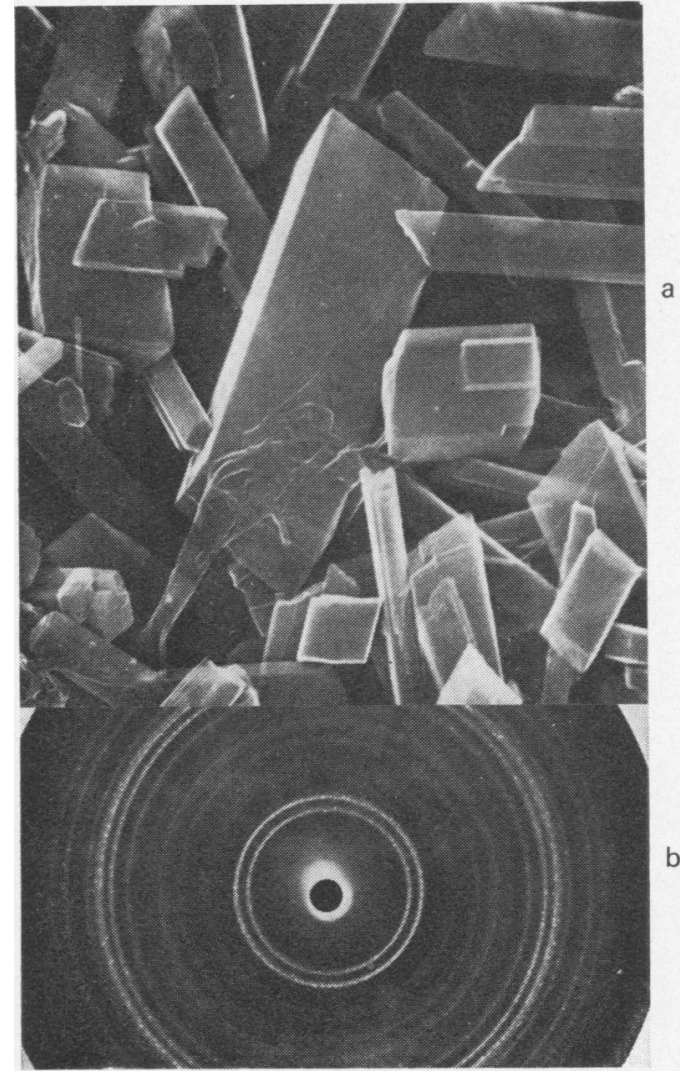

Fig. 5 (a) CPPD crystals isolated from $L 4 / 5$ disc shown in Fig. 4 (Scanning electron micrograph $\times 14$ 000). (b) $X$-ray diffraction pattern of crystals shown in (a). (Courtesy of Dr D.W. L. Hukins.)

known. That the deposits may consist of calcium pyrophosphate dihydrate (CPPD) is a relatively recent discovery (McCarty and Gatter, 1964; Bywaters et al., 1971). Such deposits are a local manifestation of CPPD deposition disease ("chondrocalcinosis') and may lead to severe disc degeneration (Sit'aj and Zitnan, 1967). Fig. 4 shows the clinical radiograph of the lower lumbar spine (interpreted as disc degeneration) in a man aged 49 on whom a discectomy was performed for backache and sciatica but who was otherwise symptomless. Fragments of the $L 4$ disc contained crystals (Fig. 5a) identified as CPPD by $x$-ray diffraction (Fig. 5b).

I mention this now fairly well-known condition because it has recently become obvious to me that the pathological examination of discectomy specimens may provide the first evidence of CPPD deposition disease. This in turn requires that the patient be screened for other occult conditions such as primary hyperparathyroidism which seem to be associated with deposition of CPPD (Hamilton, 1978). Thus the pathological diagnosis of CPPD in discs is clinically important.

\section{References}

Adams, P., Eyre, D. R., and Muir, H. (1977). Biochemical aspects of development and ageing of human lumbar intervertebral discs. Rheumatology and Rehabilitation, 16, 22-29.

Ball, J. (1971). Enthesopathy of rheumatoid and ankylosing spondylitis. Annals of the Rheumatic Diseases, 30, 213-223.

Ball, J., and Sharp, J. (1971). Rheumatoid arthritis of the cervical spine. In Modern Trends in Rheumatology-2, edited by A. G. S. Hill, p. 117. Butterworth, London.

Bijlsma, F., and Copius Peereboom, J. W. (1972). The ageing pattern of human intervertebral disc. Gerontologia, 18, 157-168.

Bywaters, E. G. L., Hamilton, E. B. D., and Williarns, R. (1971). The spine in idiopathic haemochromatosis. Annals of the Rheumatic Diseases, 30, 453-465.

Cawley, M. I. D., Chalmers, T. M., Kellgren, J. H., and Ball, J. (1972). Destructive lesions of vertebral bodies in ankylosing spondylitis. Annals of the Rheumatic Diseases, 31, 345-358.

Farfan, H. F. (1977). A reorientation in the surgical approach to degenerative lumbar intervertebral joint disease. Orthopedic Clinics of North America, 8, 9-21.

Gower, W. E., and Pedrini, V. (1969). Age-related variations in proteinpolysaccharides from human nucleus pulposus, annulus fibrosus, and costal cartilage. Journal of Bone and Joint Surgery, 51-A, 1154-1162.

Hamilton. E. B. D. (1978). Other metabolic arthropathies In Copeman's Textbook of the Rheumatic Diseases, 5th edition, edited by J. T. Scott, pp. 692-706. Churchill Livingstone, Edinburgh and London.

Hilton, R. C., Ball, J., and Benn, R. T. (1976). Vertebral end-plate lesions (Schmorl's nodes) in the dorsolumbar spine. Annals of the Rheumatic Diseases, 35, 127-131.

Hilton, R. C., Ball, J., and Benn, R. T. (1977). In-vitro studies of spinal mobility and its relation to disc degeneration and osteoarthrosis of zygapophyseal joints. Communication to the Society for Back Pain Research.

Jayson, M. I. V., Herbert, C. M., and Barks, J. S. (1973). Intervertebral discs: nuclear morphology and bursting pressures. Annals of the Rheumatic Diseases, 32, 308315.

Kellgren, J. H. (1977). The anatomical source of back pain. Rheumatology and Rehabilitation, 16, 3-12.

Lawrence, J. S. (1977). Rheumatism in Populations. Heinemann, London.

Markolf, K. L. (1972). Deformation of the thoracolumbar intervertebral joints in response to external loads. Journal of Bone and Joint Surgery, 54-A, 511-533.

McCarty, D. J., Jr., and Gatter, R. A. (1964). Pseudogout syndrome (articular chondrocalcinosis). In Bulletin on 
the Rheumatic Diseases, edited by J. J. Bunin, 14, 331-334.

Nachemson, A. (1960). Lumbar intradiscal pressure. Experimental studies on post mortem material. Acta Orthopaedica Scandinavica, Suppl. 43.

Nachemson, A. (1975). Towards a better understanding of low-back pain: A review of the mechanics of the lumbar disc. Rheumatology and Rehabilitation, 14, 129-143.

Naylor, A., Happey, F., Turner, R. 'L., Shentall, R. D., West, D. C., and Richardson, C. '(1975). Enzymic and immunological activity in the intervertebral disc.
Orthopedic Clinics of North America, 6, 51-58.

Punjabi, M. M. (1977). Experimental determination of spinal motion segment behaviour. Orthopedic Clinics of North America, 8, 169-180.

Sit'aj, S., and Zitnan, D. (1967). Spinal changes in chondrocalcinosis. In Proceedings of the Sixth European Congress of Rheumatology, 547-558.

Vernon-Roberts, B., and Pirie. C. J. (1973). Healing trabecular microfractures in the bodies of lumbar vertebrae. Annals of the Rheumatic Diseases, 32, 127-412. 